\title{
Non-linear mechanical, electrical and thermal phenomena in piezoelectric crystals
}

\author{
F.Warkusz, A.Linek \\ Institute of Physics, Opole University, 45-052 Opole, Poland \\ Received August 25, 2002, in final form March 31, 2003

\begin{abstract}
Mechanical, electrical and thermal phenomena occurring in piezoelectric crystals were examined by non-linear approximation. For this purpose, use was made of the thermodynamic function of state, which describes an anisotropic body. Considered was the Gibbs function. The calculations included strain tensor $\varepsilon_{i j}=f\left(\sigma_{k l}, E_{n}, T\right)$, induction vector $D_{m}=$ $f\left(\sigma_{k l}, E_{n}, T\right)$ and entropy $S=f\left(\sigma_{k l}, E_{n}, T\right)$ as function of stress $\sigma_{k l}$, field strength $E_{n}$ and temperature difference $T$. The equations obtained apply to anisotropic piezoelectric bodies provided that the "forces" $\sigma_{k l}, E_{n}, T$ acting on the crystal are known.
\end{abstract}

Key words: piezoelectric crystals, thermodynamics, anisotropic bodies, tensors

PACS: $65.40 .-b, 77.65 .-j, 77.65 . B n, 77.65 . L y, 05.70 . C e$

\section{Introduction}

The present paper provides a thermodynamic description of elastic (mechanical), electrical and thermal properties of crystals in a non-linear approximation. The physical processes and phenomena that occur in a real crystal generally are of a non-linear character, similarly to those in any other physical body which displays a certain degree of nonlinearity. It is conventional to describe non-linear properties (i.e., physical nonlinearity) in terms of higher-order material constants, and such are the factors of proportionality incorporated in the Taylor series expansion of a non-linear relation describing the given effect. In general, the mentioned constants are tensors.

Nonlinearity manifests in the values of the second-order material constants being the functions of the values of the applied forces which act onto the crystal, e.g. the elasticity constants can be a function of the applied stress $\sigma$. Thus, the standard Hooke equation may be written as follows:

$$
\varepsilon=s(\sigma) \sigma
$$


where $\varepsilon$ denotes strain and $s(\sigma)$ is an elasticity constant which varies according to the stress $\sigma$ applied. Hence, $s(\sigma)$ takes the form

$$
s(\sigma)=s_{1}+s_{2} \sigma+s_{3} \sigma^{2}+\ldots,
$$

where $s_{1}$ is a second-order material constant (modulus of elasticity).

By linear approximation, equation (1.1) becomes as follows: $\varepsilon=s_{1} \sigma$, where $s_{1}=\partial \varepsilon / \partial \sigma$.

Using non-linear approximation, we have

$$
\varepsilon=\frac{\partial \varepsilon}{\partial \sigma} \sigma+\frac{1}{2 !} \frac{\partial^{2} \varepsilon}{\partial \sigma^{2}} \sigma^{2}+\frac{1}{3 !} \frac{\partial^{3} \varepsilon}{\partial \sigma^{3}} \sigma^{3}+\ldots
$$

where

$$
s_{1}=\frac{\partial \varepsilon}{\partial \sigma}, \quad s_{2}=\frac{1}{2 !} \frac{\partial^{2} \varepsilon}{\partial \sigma^{2}}, \quad s_{3}=\frac{1}{3 !} \frac{\partial^{3} \varepsilon}{\partial \sigma^{3}} .
$$

The coefficients $s_{1}, s_{2}, s_{3}, \ldots$ of equation (1.2) can be regarded as material constants which are proportional to the stresses $(\sigma)$ of relevant powers. Hence, they are material constants of second, third, fourth or higher order. Thus, the Hooke equation for non-linear effects can be written as $[1,2]$

$$
\varepsilon=s(\sigma) \sigma=s_{1} \sigma+s_{2} \sigma^{2}+s_{3} \sigma^{3}+\ldots \quad \text { or } \quad \varepsilon=\left(s_{1}+s_{2} \sigma+s_{3} \sigma^{2}+\ldots\right) \sigma .
$$

With tensor notation for anisotropic bodies, the relation of

$$
s=s_{1}+s_{2} \sigma+s_{3} \sigma^{2}+\ldots
$$

takes the following form:

$$
s=s_{i j k l}+s_{i j k l p q} \sigma_{p q}+s_{i j k l p q r s} \sigma_{p q} \sigma_{r s}+\ldots
$$

and the Hooke equation

$$
\varepsilon=s(\sigma) \sigma=s_{1} \sigma+s_{2} \sigma^{2}+s_{3} \sigma^{3}+\ldots
$$

becomes

$$
\varepsilon_{i j}=s_{i j k l} \sigma_{k l}+s_{i j k l p q} \sigma_{p q} \sigma_{k l}+s_{i j k l p q r s} \sigma_{p q} \sigma_{r s} \sigma_{k l}+\ldots
$$

in tensor notation, where $s_{i j k l}$ is a fourth-order tensor (second-order material constant), $s_{i j k l p q}$ is a sixth-order tensor (third-order material constant), $s_{i j k l p q r s}$ is an eight-order tensor (fourth-order material constant), and the coefficients $i, j, k, l, p$, $q, r, s$ take the values of $1,2,3$. Summation signs have been omitted [1,3,4].

Higher-order material constants are formally derived from thermodynamic functions. The procedure is similar to that for the linear case. If, for example, strain $\varepsilon$ is produced simultaneously by stress $\sigma$, by electric field $E$, and temperature variations $T$, the equation of state for non-linear processes, which includes material constants only up to the third order, can be written as

$$
\varepsilon=s_{1} \sigma+d_{1} E+\alpha_{1} T+s_{2} \sigma^{2}+d_{2} E^{2}+\alpha_{2} T^{2}+k_{\sigma E} \sigma E+k_{\sigma T} \sigma T+k_{E T} E T,
$$


where

$$
\begin{aligned}
& s_{1}=\frac{\partial \varepsilon}{\partial \sigma}, \quad d_{1}=\frac{\partial \varepsilon}{\partial E}, \quad \alpha_{1}=\frac{\partial \varepsilon}{\partial T}, \quad s_{2}=\frac{1}{2} \frac{\partial^{2} \varepsilon}{\partial \sigma^{2}}, \quad d_{2}=\frac{1}{2} \frac{\partial^{2} \varepsilon}{\partial E^{2}}, \\
& \alpha^{2}=\frac{1}{2} \frac{\partial^{2} \varepsilon}{\partial T^{2}}, \quad k_{\sigma E}=\frac{\partial^{2} \varepsilon}{\partial \sigma \partial E}, \quad k_{\sigma T}=\frac{\partial^{2} \varepsilon}{\partial \sigma \partial T}, \quad k_{E T}=\frac{\partial^{2} \varepsilon}{\partial E \partial T} .
\end{aligned}
$$

Apart from second-order material constants, $s_{1}, d_{1}, \alpha_{1}$, and third-order material constants $s_{2}, d_{2}, \alpha_{2}$, there are also third-order "mixed" material constants $k_{\sigma E}, k_{\sigma T}$ and $k_{E T}$. The order of the material constant is defined by the order of the derivative of the thermodynamic function and not by the order of the "force" acting onto the crystal or by the order of the material constant tensor.

The problem will be presented more in detail in section 2 .

\section{Thermodynamic relations in crystals and Gibbs functions}

According to the first law of thermodynamics, the total energy $U$ of a body is the sum of different energy types. In piezoelectric crystals, energy balance is primarily accounted for by mechanical, electrical and thermal energy. The effect of magnetic energy or gravitational energy on the phenomena occurring in piezoelectric crystals may be neglected. In general, there are eight thermodynamic functions that can be used to describe a piezoelectric phenomenon. The form of the function depends on the choice of the independent variables, selected according to the conditions under which the crystal is to be examined [2].

In the present paper we confine ourselves to thermodynamics, that is, Gibbs function $G$. Mathematical analysis enables us to define particular material constants, as well as to establish many interesting relations between them, by linear and nonlinear approximation.

$$
G=U-\varepsilon_{i j} \sigma_{i j}-E_{m} D_{m}-T S
$$

where $i, j, m=1,2,3$.

Let us consider the differential form $\mathrm{d} G$, which describes the state of the crystal following the application of three different "forces" - stress $\sigma_{i j}$, electric field $E_{m}$, and temperature $T$. The forces acting onto the crystal are independent variables. Thus, we have

$$
\mathrm{d} G=\mathrm{d} U-\varepsilon_{i j} \mathrm{~d} \sigma_{i j}-\sigma_{i j} \mathrm{~d} \varepsilon_{i j}-E_{m} \mathrm{~d} D_{m}-D_{m} \mathrm{~d} E_{m}-S \mathrm{~d} T-T \mathrm{~d} S .
$$

By virtue of the first and second law of thermodynamics,

$$
\mathrm{d} U=\sigma_{i j} \mathrm{~d} \varepsilon_{i j}+E_{m} \mathrm{~d} D_{m}+T \mathrm{~d} S,
$$

where $i, j, m=1,2,3$.

Substituting (2.3) into (2.2), we obtain

$$
\mathrm{d} G=-\varepsilon_{i j} \mathrm{~d} \sigma_{i j}-D_{m} \mathrm{~d} E_{m}-S \mathrm{~d} T .
$$


Strain $\varepsilon_{i j}$, induction $D_{m}$ and entropy $S$ are functions of stress $\sigma_{k l}$, electric field $E_{n}$ and temperature $T$ :

$$
\varepsilon_{i j}=f\left(\sigma_{k l}, E_{n}, T\right), \quad D_{m}=f\left(\sigma_{k l}, E_{n}, T\right), \quad S=f\left(\sigma_{k l}, E_{n}, T\right) .
$$

Differentiating the function $G$ with respect to individual independent variables, and having determined the remaining values, we obtain

$$
\mathrm{d} G=\left(\frac{\partial G}{\partial \sigma_{i j}}\right)_{E, T} \mathrm{~d} \sigma_{i j}+\left(\frac{\partial G}{\partial E_{m}}\right)_{\sigma, T} \mathrm{~d} E_{m}+\left(\frac{\partial G}{\partial T}\right)_{\sigma, E} \mathrm{~d} T .
$$

Comparing the coefficients of (2.4) and (2.5), we can derive the relations that describe particular quantities:

$$
\varepsilon_{i j}=-\left.\frac{\partial G}{\partial \sigma_{i j}}\right|_{E, T}, \quad D_{m}=-\left.\frac{\partial G}{\partial E_{m}}\right|_{\sigma, T}, \quad S=-\left.\frac{\partial G}{\partial T}\right|_{\sigma, E} .
$$

The indices in the lower part of the vertical line show the independent variable, which takes a constant value during differentiation.

The function $\varepsilon_{i j}, D_{m}$ and $S$ can be expanded into a Taylor series. All derivatives incorporated in the Taylor's series are the material constants.

\subsection{Material constants derived from function $\varepsilon_{i j}$}

Second-order elasticity constant

$$
\left.\frac{\partial \varepsilon_{i j}}{\partial \sigma_{k l}}\right|_{E, T}=\frac{\partial}{\partial \sigma_{k l}}\left(-\frac{\partial G}{\partial \sigma_{i j}}\right)=-\left.\frac{\partial^{2} G}{\partial \sigma_{k l} \partial \sigma_{i j}}\right|_{E, T}=s_{i j k l}^{E, T} .
$$

Second-order piezoelectric constant

$$
\left.\frac{\partial \varepsilon_{i j}}{\partial E_{n}}\right|_{\sigma, T}=\frac{\partial}{\partial E_{n}}\left(-\frac{\partial G}{\partial \sigma_{i j}}\right)=-\left.\frac{\partial^{2} G}{\partial E_{n} \partial \sigma_{i j}}\right|_{T}=d_{i j n}^{T} .
$$

Second-order piezocalorific constant

$$
\left.\frac{\partial \varepsilon_{i j}}{\partial T}\right|_{\sigma, E}=\frac{\partial}{\partial T}\left(-\frac{\partial G}{\partial \sigma_{i j}}\right)=-\left.\frac{\partial^{2} G}{\partial T \partial \sigma_{i j}}\right|_{E}=\alpha_{i j}^{E} .
$$

Third-order elasticity constant

$$
\left.\frac{\partial^{2} \varepsilon_{i j}}{\partial \sigma_{k l} \partial \sigma_{p q}}\right|_{E, T}=\frac{\partial^{2}}{\partial \sigma_{k l} \partial \sigma_{p q}}\left(-\frac{\partial G}{\partial \sigma_{i j}}\right)=-\left.\frac{\partial^{3} G}{\partial \sigma_{k l} \partial \sigma_{p q} \partial \sigma_{i j}}\right|_{E, T}=s_{i j k l p q}^{E, T} .
$$

Third-order piezoelectric constant

$$
\left.\frac{\partial^{2} \varepsilon_{i j}}{\partial \sigma_{k l} \partial E_{n}}\right|_{T}=\frac{\partial^{2}}{\partial \sigma_{k l} \partial E_{n}}\left(-\frac{\partial G}{\partial \sigma_{i j}}\right)=-\left.\frac{\partial^{3} G}{\partial \sigma_{k l} \partial E_{n} \partial \sigma_{i j}}\right|_{T}=d_{i j k l n}^{T} .
$$


Third-order piezocalorific constant

$$
\left.\frac{\partial^{2} \varepsilon_{i j}}{\partial \sigma_{k l} \partial T}\right|_{E}=\frac{\partial^{2}}{\partial \sigma_{k l} \partial T}\left(-\frac{\partial G}{\partial \sigma_{i j}}\right)=-\left.\frac{\partial^{3} G}{\partial \sigma_{k l} \partial T \partial \sigma_{i j}}\right|_{E}=\alpha_{i j k l}^{E} .
$$

Third-order electrostriction constant

$$
\left.\frac{\partial^{2} \varepsilon_{i j}}{\partial E_{n} \partial E_{t}}\right|_{\sigma, T}=\frac{\partial^{2}}{\partial E_{n} \partial E_{t}}\left(-\frac{\partial G}{\partial \sigma_{i j}}\right)=-\left.\frac{\partial^{3} G}{\partial E_{n} \partial E_{t} \partial \sigma_{i j}}\right|_{T}=o_{i j n t}^{T} .
$$

Third-order constant

$$
\left.\frac{\partial^{2} \varepsilon_{i j}}{\partial E_{n} \partial T}\right|_{\sigma}=\frac{\partial^{2}}{\partial E_{n} \partial T}\left(-\frac{\partial G}{\partial \sigma_{i j}}\right)=-\frac{\partial^{3}}{\partial E_{n} \partial T \partial \sigma_{i j}}=k_{i j n} .
$$

Third-order thermal expansion constant

$$
\left.\frac{\partial^{2} \varepsilon_{i j}}{\partial T \partial T_{1}}\right|_{\sigma, E}=\frac{\partial^{2}}{\partial T \partial T_{1}}\left(-\frac{\partial G}{\partial \sigma_{i j}}\right)=-\left.\frac{\partial^{3} G}{\partial T \partial T_{1} \partial \sigma_{i j}}\right|_{E}=r_{i j}^{E} .
$$

\subsection{Material constants derived from function $D_{m}$}

Second-order piezoelectric constant

$$
\left.\frac{\partial D_{m}}{\partial \sigma_{k l}}\right|_{E, T}=\frac{\partial}{\partial \sigma_{k l}}\left(-\frac{\partial G}{\partial E_{m}}\right)=-\left.\frac{\partial^{2} G}{\partial \sigma_{k l} \partial E_{m}}\right|_{T}=d_{k l m}^{T} .
$$

Second-order permittivity constant

$$
\left.\frac{\partial D_{m}}{\partial E_{n}}\right|_{\sigma, T}=\frac{\partial}{\partial E_{n}}\left(-\frac{\partial G}{\partial E_{m}}\right)=-\left.\frac{\partial^{2} G}{\partial E_{n} \partial E_{m}}\right|_{\sigma, T}=\varepsilon_{m n}^{\sigma, T} .
$$

Second-order electric heat constant

$$
\left.\frac{\partial D_{m}}{\partial T}\right|_{\sigma, E}=\frac{\partial}{\partial T}\left(-\frac{\partial G}{\partial E_{m}}\right)=-\left.\frac{\partial^{2} G}{\partial T \partial E_{m}}\right|_{\sigma}=p_{m}^{\sigma} .
$$

Third-order piezoelectric constant

$$
\left.\frac{\partial^{2} D_{m}}{\partial \sigma_{k l} \partial \sigma_{p q}}\right|_{E, T}=\frac{\partial^{2}}{\partial \sigma_{k l} \partial \sigma_{p q}}\left(-\frac{\partial G}{\partial E_{m}}\right)=-\left.\frac{\partial^{3} G}{\partial \sigma_{k l} \partial \sigma_{p q} \partial E_{m}}\right|_{T}=d_{k l p q m}^{T} .
$$

Third-order electrostriction constant

$$
\left.\frac{\partial^{2} D_{m}}{\partial \sigma_{k l} \partial E_{n}}\right|_{T}=\frac{\partial^{2}}{\partial \sigma_{k l} \partial E_{n}}\left(-\frac{\partial G}{\partial E_{m}}\right)=-\left.\frac{\partial^{3} G}{\partial \sigma_{k l} \partial E_{n} \partial E_{m}}\right|_{T}=o_{k l m n}^{T} .
$$

Third-order constant

$$
\left.\frac{\partial^{2} D_{m}}{\partial \sigma_{k l} \partial T}\right|_{E}=\frac{\partial^{2}}{\partial \sigma_{k l} \partial T}\left(-\frac{\partial G}{\partial E_{m}}\right)=-\frac{\partial^{3}}{\partial \sigma_{k l} \partial T \partial E_{m}}=k_{k l m} .
$$


Third-order permittivity constant

$$
\left.\frac{\partial^{2} D_{m}}{\partial E_{n} \partial E_{t}}\right|_{\sigma, T}=\frac{\partial^{2}}{\partial E_{n} \partial E_{t}}\left(-\frac{\partial G}{\partial E_{m}}\right)=-\left.\frac{\partial^{3} G}{\partial E_{n} \partial E_{t} \partial E_{m}}\right|_{\sigma, T}=\varepsilon_{m n t}^{\sigma, T} .
$$

Third-order electric heat constant

$$
\left.\frac{\partial^{2} D_{m}}{\partial E_{n} \partial T}\right|_{\sigma}=\frac{\partial^{2}}{\partial E_{n} \partial T}\left(-\frac{\partial G}{\partial E_{m}}\right)=-\left.\frac{\partial^{3} G}{\partial E_{n} \partial T \partial E_{m}}\right|_{\sigma}=p_{m n}^{\sigma} .
$$

Third-order pyroelectric constant

$$
\left.\frac{\partial^{2} D_{m}}{\partial T_{1} \partial T}\right|_{\sigma, E}=\frac{\partial^{2}}{\partial T_{1} \partial T}\left(-\frac{\partial G}{\partial E_{m}}\right)=-\left.\frac{\partial^{3} G}{\partial T_{1} \partial T \partial E_{m}}\right|_{\sigma}=u_{m}^{\sigma} .
$$

\subsection{Material constants derived from function $S$}

Second-order piezocalorific constant

$$
\left.\frac{\partial S}{\partial \sigma_{k l}}\right|_{E, T}=\frac{\partial}{\partial \sigma_{k l}}\left(-\frac{\partial G}{\partial T}\right)=-\left.\frac{\partial^{2} G}{\partial \sigma_{k l} \partial T}\right|_{E}=\alpha_{k l}^{E} .
$$

Second-order electric heat constant

$$
\left.\frac{\partial S}{\partial E_{n}}\right|_{\sigma, T}=\frac{\partial}{\partial E_{n}}\left(-\frac{\partial G}{\partial T}\right)=-\left.\frac{\partial^{2} G}{\partial E_{n} \partial T}\right|_{\sigma}=p_{n}^{\sigma} .
$$

Second-order thermal capacity constant

$$
\left.\frac{\partial S}{\partial T}\right|_{\sigma, E}=\frac{\partial}{\partial T}\left(-\frac{\partial G}{\partial T_{1}}\right)=-\left.\frac{\partial^{2} G}{\partial T \partial T_{1}}\right|_{\sigma, E}=\xi^{\sigma, E}
$$

Third-order piezocalorific constant

$$
\left.\frac{\partial^{2} S}{\partial \sigma_{k l} \partial \sigma_{p q}}\right|_{E, T}=\frac{\partial^{2}}{\partial \sigma_{k l} \partial \sigma_{p q}}\left(-\frac{\partial G}{\partial T}\right)=-\left.\frac{\partial^{3} G}{\partial \sigma_{k l} \partial \sigma_{p q} \partial T}\right|_{E}=\alpha_{k l p q}^{E} .
$$

Third-order constant

$$
\left.\frac{\partial^{2} S}{\partial \sigma_{k l} \partial E_{n}}\right|_{T}=\frac{\partial^{2}}{\partial \sigma_{k l} \partial E_{n}}\left(-\frac{\partial G}{\partial T}\right)=-\frac{\partial^{3}}{\partial \sigma_{k l} \partial E_{n} \partial T}=k_{k l n} .
$$

Third-order thermal expansion constant

$$
\left.\frac{\partial^{2} S}{\partial \sigma_{k l} \partial T}\right|_{E}=\frac{\partial^{2}}{\partial \sigma_{k l} \partial T}\left(-\frac{\partial G}{\partial T_{1}}\right)=-\left.\frac{\partial^{3} G}{\partial \sigma_{k l} \partial T \partial T_{1}}\right|_{E}=r_{k l}^{E} .
$$

Third-order electric heat constant

$$
\left.\frac{\partial^{2} S}{\partial E_{n} \partial E_{t}}\right|_{\sigma, T}=\frac{\partial^{2}}{\partial E_{n} \partial E_{t}}\left(-\frac{\partial G}{\partial T}\right)=-\left.\frac{\partial^{3} G}{\partial E_{n} \partial E_{t} \partial T}\right|_{\sigma}=p_{n t}^{\sigma} .
$$


Third-order pyroelectric constant

$$
\left.\frac{\partial^{2} S}{\partial E_{n} \partial T}\right|_{\sigma}=\frac{\partial^{2}}{\partial E_{n} \partial T}\left(-\frac{\partial G}{\partial T_{1}}\right)=-\left.\frac{\partial^{3} G}{\partial E_{n} \partial T \partial T_{1}}\right|_{\sigma}=u_{n}^{\sigma} .
$$

Third-order thermal capacity constant

$$
\left.\frac{\partial^{2} S}{\partial T \partial T_{1}}\right|_{\sigma, E}=\frac{\partial^{2}}{\partial T \partial T_{1}}\left(-\frac{\partial G}{\partial T_{2}}\right)=-\left.\frac{\partial^{3} G}{\partial T \partial T_{1} \partial T_{2}}\right|_{\sigma, E}=\rho^{\sigma, E} .
$$

\subsection{Equalities between material constants}

$$
\begin{aligned}
& \left.\frac{\partial D_{m}}{\partial \sigma_{k l}}\right|_{E, T}=\left.\frac{\partial \varepsilon_{k l}}{\partial E_{m}}\right|_{\sigma, T}=d_{k l m}^{T},\left.\quad \frac{\partial^{2} S}{\partial \sigma_{k l} \partial \sigma_{p q}}\right|_{E, T}=\left.\frac{\partial^{2} \varepsilon_{k l}}{\partial \sigma_{p q} \partial T}\right|_{E}=\alpha_{k l p q}^{E}, \\
& \left.\frac{\partial D_{m}}{\partial \sigma_{k l} \partial \sigma_{p q}}\right|_{E, T}=\left.\frac{\partial^{2} \varepsilon_{k l}}{\partial \sigma_{p q} \partial E_{m}}\right|_{T}=d_{k l m p q}^{T},\left.\quad \frac{\partial^{2} S}{\partial E_{n} \partial T}\right|_{\sigma}=\left.\frac{\partial D_{n}}{\partial T \partial T_{1}}\right|_{\sigma, E}=u_{n}^{\sigma}, \\
& \left.\frac{\partial S}{\partial E_{n}}\right|_{\sigma, T}=\left.\frac{\partial D_{n}}{\partial T}\right|_{\sigma, E}=p_{n}^{\sigma},\left.\quad \frac{\partial^{2} S}{\partial \sigma_{k l} \partial T}\right|_{E}=\left.\frac{\partial^{2} \varepsilon_{k l}}{\partial T \partial T_{1}}\right|_{\sigma, E}=r_{k l}^{E}, \\
& \left.\frac{\partial^{2} S}{\partial E_{n} \partial E_{t}}\right|_{\sigma, T}=\left.\frac{\partial D_{n}}{\partial E_{t} \partial T}\right|_{\sigma}=p_{n t}^{\sigma},\left.\quad \frac{\partial D_{m}}{\partial \sigma_{k l} \partial E_{n}}\right|_{T}=\left.\frac{\partial^{2} \varepsilon_{k l}}{\partial E_{n} \partial E_{m}}\right|_{\sigma, T}=o_{k l m n}^{T}, \\
& \left.\frac{\partial S}{\partial \sigma_{k l}}\right|_{E, T}=\left.\frac{\partial \varepsilon_{k l}}{\partial T}\right|_{\sigma, E}=\alpha_{k l}^{E},\left.\quad \frac{\partial^{2} \varepsilon_{i j}}{\partial E_{n} \partial T}\right|_{E}=\left.\frac{\partial^{2} D_{n}}{\partial \sigma_{i j} \partial T}\right|_{E}=\left.\frac{\partial^{2} S}{\partial \sigma_{i j} \partial E_{n}}\right|_{T}=k_{i j n} .
\end{aligned}
$$

With the material constants (tensors) defined above, we can rewrite function $\varepsilon_{i j}$, $D_{m}$ and $S$ from Taylor series into form:

$$
\begin{aligned}
\varepsilon_{i j}= & \left(s_{i j k l}^{E, T}+\frac{1}{2} s_{i j k l p q}^{E, T} \sigma_{p q}+\frac{1}{2} d_{i j k l n}^{T} E_{n}+\frac{1}{2} \alpha_{i j k l}^{E} T\right) \sigma_{k l} \\
& +\left(d_{i j n}^{T}+\frac{1}{2} d_{k l i j n}^{T} \sigma_{k l}+\frac{1}{2} o_{i j n t}^{T} E_{t}+\frac{1}{2} k_{i j n} T\right) E_{n} \\
& +\left(\alpha_{i j}^{E}+\frac{1}{2} \alpha_{i j k l}^{E} \sigma_{k l}+\frac{1}{2} k_{i j n} E_{n}+\frac{1}{2} r_{i j}^{E} T_{1}\right) T \\
D_{m}= & \left(d_{m k l}^{T}+\frac{1}{2} d_{m k l p q}^{T} \sigma_{p q}+\frac{1}{2} k_{m k l} T+\frac{1}{2} o_{k l m n}^{T} E_{n}\right) \sigma_{k l} \\
& +\left(\varepsilon_{n m}^{T, \sigma}+\frac{1}{2} \varepsilon_{n m t}^{T, \sigma} E_{t}+\frac{1}{2} o_{n m k l}^{T} \sigma_{k l}+\frac{1}{2} p_{n m}^{\sigma} T\right) E_{n} \\
& +\left(p_{m}^{\sigma}+\frac{1}{2} k_{m k l} \sigma_{k l}+\frac{1}{2} p_{m n}^{\sigma} E_{n}+\frac{1}{2} u_{m}^{\sigma} T_{1}\right) T \\
S= & \left(\alpha_{k l}^{E}+\frac{1}{2} \alpha_{k l p q}^{E} \sigma_{p q}+\frac{1}{2} k_{k l n} E_{n}+\frac{1}{2} r_{k l}^{E} T\right) \sigma_{k l} \\
& +\left(p_{n}^{\sigma}+\frac{1}{2} k_{n k l} \sigma_{k l}+\frac{1}{2} p_{n t}^{\sigma} E_{t}+\frac{1}{2} u_{n}^{\sigma} T\right) E_{n} \\
& +\left(\xi^{E, \sigma}+\frac{1}{2} r_{k l}^{E} \sigma_{k l}+\frac{1}{2} u_{n}^{\sigma} E_{n}+\rho^{E, \sigma} T_{1}\right) T .
\end{aligned}
$$




\section{Linear and non-linear effects of the equations of state}

Considering, for example, the equation of state derived from the Gibbs function $G$ for non-linear effects, we can easily obtain the equations of state for linear effects by neglecting higher-order tensors. Thus, by virtue of (2.6), (2.7) and (2.8) we have

$$
\begin{aligned}
\varepsilon_{i j} & =s_{i j k l}^{E, T} \sigma_{k l}+d_{i j n}^{T} E_{n}+\alpha_{i j}^{E} T, \\
D_{m} & =d_{m k l}^{T} \sigma_{k l}+\varepsilon_{n m}^{T, \sigma} E_{n}+p_{m}^{\sigma} T \\
S & =\alpha_{k l}^{E} \sigma_{k l}+p_{n}^{\sigma} E_{n}+\xi^{E, \sigma} T
\end{aligned}
$$

which are valid for linear effects $[7,8,9]$.

By virtue of the symmetry of some tensors (tensor components in matrix tables), the relations of (3.1) can be presented in matrix form.

Hooke's law is often expressed in its contracted notation. Then, the equivalence between the components of the compliance fourth-rank tensor $s_{i j k l}$ and the components of the $6 \times 6$ matrix $s$ is shown to be:

$$
\begin{aligned}
& s_{i j k l}=s_{m n}, \quad m, n=1,2,3, \\
& 2 s_{i j k l}=s_{m n}, \quad m=1,2,3, n=4,5,6, \\
& 4 s_{i j k l}=s_{m n}, \quad m, n=4,5,6
\end{aligned}
$$

in which the following contraction rule is applied for replacing a pair of indices by a single contracted index: $11 \rightarrow 1,22 \rightarrow 2,33 \rightarrow 3,(23,32) \rightarrow 4,(13,31) \rightarrow 5,(12$, 21) $\rightarrow 6$.

Furthermore, the full tensor suffixes of the stresses $\sigma$ and strains $\varepsilon$ are contracted according to the scheme:

$$
\begin{aligned}
& \sigma_{11}=\sigma_{1}, \quad \sigma_{22}=\sigma_{2}, \quad \sigma_{33}=\sigma_{3}, \\
& \left(\sigma_{23}, \sigma_{32}\right)=\sigma_{4}, \quad\left(\sigma_{13}, \sigma_{31}\right)=\sigma_{5}, \quad\left(\sigma_{12}, \sigma_{21}\right)=\sigma_{6}, \\
& \varepsilon_{11}=\varepsilon_{1}, \quad \varepsilon_{22}=\varepsilon_{2}, \quad \varepsilon_{33}=\varepsilon_{3}, \\
& \left(2 \varepsilon_{23}, 2 \varepsilon_{32}\right)=\varepsilon_{4}, \quad\left(2 \varepsilon_{13}, 2 \varepsilon_{31}\right)=\varepsilon_{5}, \quad\left(2 \varepsilon_{12}, 2 \varepsilon_{21}\right)=\varepsilon_{6}
\end{aligned}
$$

and

$$
\begin{array}{r}
d_{i j k}=d_{m n}, \quad m, n=1,2,3, \\
2 d_{i j k}=d_{m n}, \quad m=1,2,3, n=4,5,6, \\
\alpha_{11}=\alpha_{1}, \quad \alpha_{22}=\alpha_{2}, \quad \alpha_{33}=\alpha_{3}, \\
\left(\alpha_{23}, \alpha_{32}\right)=\alpha_{4}, \quad\left(\alpha_{13}, \alpha_{31}\right)=\alpha_{5}, \quad\left(\alpha_{12}, \alpha_{21}\right)=\alpha_{6} .
\end{array}
$$

The relations of (3.1) constitute the starting set of the equations of state, which describe the environment, and they are widely used to solve the problems dealt with in piezoelectricity. Basic effects are described by the material constants (tensors) $s_{i j k l}^{E, T}, \varepsilon_{n m}^{T, \sigma}, \xi^{E, \sigma}$ that occur at the diagonal of the set of equations, whereas conjugate 
effects are defined by the remaining constants. On rewriting the material constants of (3.1) in a matrix form, we obtain a symmetrical matrix of linear effects:

$$
\left[\begin{array}{c}
\varepsilon_{i j} \\
D_{m} \\
S
\end{array}\right]=\left[\begin{array}{ccc}
s_{i j k l}^{E, T} & d_{i j n}^{T} & \alpha_{i j}^{E} \\
d_{m k l}^{T} & \varepsilon_{n m}^{T, \sigma} & p_{m}^{\sigma} \\
\alpha_{k l}^{E} & p_{n}^{\sigma} & \xi^{E, \sigma}
\end{array}\right]\left[\begin{array}{c}
\sigma_{k l} \\
E_{n} \\
T
\end{array}\right] .
$$

The equivalence between the components of the compliance sixth-rank tensor $s_{i j k l p q}$ and the components of the $6 \times 21$ matrix is shown to be:

$$
\begin{array}{rll}
S_{i j k l p q}=s_{m n o}, & & m=1,2,3, n=o=1,2,3, \\
2 s_{i j k l p q}=s_{m n o}, & & m=1,2,3, n \neq o, n=1,2, o=2,3, \\
4 s_{i j k l p q}=s_{m n o}, & & m=1,2,3, n=1,2,3, o=4,5,6, n=o=4,5,6, \\
8 s_{i j k l p q}=s_{m n o}, & & m=1,2,3, n=4,5, o=5,6, \\
2 s_{i j k l p q}=s_{m n o}, & & m=4,5,6, n=o=1,2,3, \\
4 s_{i j k l p q}=s_{m n o}, & & m=4,5,6, n \neq o, n=1,2, o=2,3, \\
8 s_{i j k l p q}=s_{m n o}, & & m=4,5,6, n=1,2,3, o=4,5,6, n=o=4,5,6, \\
16 s_{i j k l p q}=s_{m n o}, & & m=4,5,6, n=4,5, o=5,6 .
\end{array}
$$

The equation

$$
\varepsilon_{i j}=\frac{1}{2} s_{i j k l p q}^{E T} \sigma_{k l} \sigma_{p q}, \quad i, j, k, l, p, q=1,2,3
$$

can be written in the form

$$
\varepsilon_{m}=\frac{1}{2} s_{m n o} \sigma_{n} \sigma_{o}, \quad m, n, o=1,2,3, \ldots
$$

Considering equations (2.6), (2.7) and (2.8) we obtain in a non-linear approximation:

$$
\left.\begin{array}{r}
\varepsilon_{i j}=s_{i j k l} \sigma_{k l}+\frac{1}{2} s_{i j k l p q} \sigma_{k l} \sigma_{p q}+\ldots, \\
D_{m}=d_{m k l} \sigma_{k l}+\frac{1}{2} d_{m k l p q} \sigma_{k l} \sigma_{p q}+\ldots, \\
S=\alpha_{k l} \sigma_{k l}+\frac{1}{2} \alpha_{k l p q} \sigma_{k l} \sigma_{p q}+\ldots
\end{array}\right\} \quad \text { if } E_{n}=0, \Delta T=0,
$$

An analogical matrix equation can be derived for non-linear effects (equations (2.6), (2.7) and (2.8)):

$$
\left[\begin{array}{l}
\varepsilon_{i j} \\
D_{m} \\
S
\end{array}\right]=\left[\begin{array}{ccc}
A & D & E \\
D & B & F \\
E & F & C
\end{array}\right]\left[\begin{array}{l}
\sigma_{k l} \\
E_{n} \\
T
\end{array}\right]
$$


where the basic effects are given by the following constants:

$$
\begin{aligned}
& A=s_{i j k l}^{E, T}+\frac{1}{2} s_{i j k l p q}^{E, T} \sigma_{p q}+\frac{1}{2} d_{i j k l n}^{T} E_{n}+\frac{1}{2} \alpha_{i j k l}^{E} T, \\
& B=\varepsilon_{n m t}^{T, \sigma}+\frac{1}{2} \varepsilon_{n m t}^{T, \sigma} E_{n}+\frac{1}{2} o_{n m k l}^{T} \sigma_{k l}+\frac{1}{2} p_{n m}^{\sigma} T, \\
& C=\xi^{E, \sigma}+\frac{1}{2} \rho^{E, \sigma} T+\frac{1}{2} u_{n}^{\sigma} E_{n}+\frac{1}{2} r_{k l}^{E} \sigma_{k l}
\end{aligned}
$$

and the conjugate effects are described by

$$
\begin{aligned}
D & =d_{i j n}^{T}+\frac{1}{2} d_{k l i j n}^{T} \sigma_{k l}+\frac{1}{2} o_{i j n t}^{T} E_{t}+\frac{1}{2} k_{i j n} T, \\
E & =\alpha_{i j}^{E}+\frac{1}{2} \alpha_{i j k l}^{E} \sigma_{k l}+\frac{1}{2} r_{i j}^{E} T+\frac{1}{2} k_{i j n} E_{n}, \\
F & =p_{m}^{\sigma}+\frac{1}{2} p_{m n}^{\sigma} E_{n}+\frac{1}{2} k_{m k l} \sigma_{k l}+\frac{1}{2} u_{m}^{\sigma} T .
\end{aligned}
$$

As we can see, the matrix of non-linear effects is also a symmetrical one.

\section{Summary}

Using the Gibbs function $(G)$, the equations describing the physical quantities $\varepsilon_{i j}$, $D_{m}, S$ have been derived for the non-linear effects of piezoelectric bodies. Relevant equations are included in section 3. It has been demonstrated that the relations between the material constants, which are tensors of zeroth to sixth order, can be written in non-linear approximation in the form of material equations where the elements of the matrices are the sums of appropriate matrices which represent the tensors (relation of (3.3)). The relations of (3.3) were used to derive the linear effects (relations of (3.2)).

The equations describing linear and non-linear phenomena for particular crystallographic point groups reduce because of monocrystal symmetry $[5,6,12,13,14,15,16]$.

From the rewritten relations of $(2.6,2.7,2.8)$ in the matrix notation (table 1) we can easily identify the interrelations of the tensor components, especially for separate physical effects. Further simplifications and decays of some components of higher order tensors may be obtained for appropriate point groups (crystal classes).

Table 1. Matrices of the coefficients (tensor components) in non-linear expansion.

\begin{tabular}{|c|c|c|c|c|c|c|c|c|c|}
\hline & $\sigma_{n}$ & $\sigma_{n} \sigma_{o}$ & $E_{n} \sigma_{o}$ & $\sigma_{n} T$ & $E_{n}$ & $E_{n} E_{t}$ & $E_{n} T$ & $T$ & $T^{2}$ \\
\hline \multirow{2}{*}{$\varepsilon_{m}$} & $s_{m n}$ & $s_{m n o}$ & $d_{m n o}$ & $\alpha_{m n l}$ & $d_{n m}$ & $o_{m n t}$ & $k_{m n}$ & $\alpha_{m}$ & $r_{m}$ \\
& $6 \times 6$ & $6 \times 21$ & $6 \times 15$ & $6 \times 6$ & $6 \times 3$ & $6 \times 6$ & $6 \times 3$ & $6 \times 1$ & $6 \times 1$ \\
\hline \multirow{2}{*}{$D_{m}$} & $d_{m n}$ & $d_{n m o}$ & $O_{\text {omn }}$ & $k_{n m}$ & $\varepsilon_{m n}$ & $\varepsilon_{m n t}$ & $p_{n m}$ & $p_{m}$ & $u_{m}$ \\
& $3 \times 6$ & $3 \times 21$ & $3 \times 15$ & $3 \times 6$ & $3 \times 3$ & $3 \times 6$ & $3 \times 3$ & $3 \times 1$ & $3 \times 1$ \\
\hline \multirow{2}{*}{$S$} & $\alpha_{n}$ & $\alpha_{n o}$ & $k_{\text {on }}$ & $r_{n}$ & $p_{n}$ & $p_{n t}$ & $u_{n}$ & $\xi$ & $\rho$ \\
& $1 \times 6$ & $1 \times 21$ & $1 \times 15$ & $1 \times 6$ & $1 \times 3$ & $1 \times 6$ & $1 \times 3$ & $1 \times 1$ & $1 \times 1$ \\
\hline
\end{tabular}

$(6+3+1) \times(6+21+15+6+3+6+3+1+1)=10 \times 62$ 
In 1952, with the aim to interpret the properties of the tetragonal antiferroelectric crystal $\mathrm{ND}_{4} \mathrm{D}_{2} \mathrm{PO}_{4}$, Mason $[10,11]$ made use of the elastic Gibbs function $\mathrm{G}_{1}$ :

$$
G_{1}=U-\varepsilon_{i j} \sigma_{i j}-T S
$$

in its differential form:

$$
\mathrm{d} G_{1}=-\varepsilon_{i j} \mathrm{~d} \sigma_{k l}+E_{n} \mathrm{~d} D_{n}-S \mathrm{~d} T .
$$

The equations and formulae describing $\varepsilon_{i j}, E_{n}$ and $S$, which have been derived by Mason [10], depend on $\sigma_{k l}, D_{n}$ and $T$. So it is not surprising that they differ from the equations presented in this paper (equations (2.6), (2.7) and (2.8)) both in linear and non-linear approximations. In our calculations we used a Gibbs function which had the form of $G$ :

$$
G=U-\varepsilon_{i j} \sigma_{k l}-E_{n} D_{n}-T S
$$

and a differential form of

$$
\mathrm{d} G=-\varepsilon_{i j} \mathrm{~d} \sigma_{k l}-D_{n} \mathrm{~d} E_{n}-S \mathrm{~d} T .
$$

The equations obtained for $\varepsilon_{i j}, D_{n}$ and $S$ depend on $\sigma_{k l}, E_{n}$ and $T$. There is a definite sense in which our results differ from those achieved by Mason. For example, according to Mason (function $G_{1}$ ) [10] we have the following relations for the linear effects:

$$
\begin{aligned}
\varepsilon_{i j} & =\left.\frac{\partial \varepsilon_{i j}}{\partial \sigma_{k l}}\right|_{D, T} \sigma_{k l}+\left.\frac{\partial \varepsilon_{i j}}{\partial D_{m}}\right|_{\sigma, T} D_{m}+\left.\frac{\partial \varepsilon_{i j}}{\partial T}\right|_{\sigma, D} T, \\
E_{n} & =\left.\frac{\partial E_{n}}{\partial \sigma_{k l}}\right|_{D, T} \sigma_{k l}+\left.\frac{\partial E_{n}}{\partial D_{m}}\right|_{\sigma, T} D_{m}+\left.\frac{\partial E_{n}}{\partial T}\right|_{\sigma, D} T \\
S & =\left.\frac{\partial S}{\partial \sigma_{k l}}\right|_{D, T} \sigma_{k l}+\underline{\left.\frac{\partial S}{\partial D_{m}}\right|_{\sigma, T} D_{m}}+\left.\frac{\partial S}{\partial T}\right|_{\sigma, D} T .
\end{aligned}
$$

Our results (based on function $\mathrm{G}$ ) take the form

$$
\begin{aligned}
\varepsilon_{i j} & =\left.\frac{\partial \varepsilon_{i j}}{\partial \sigma_{k l}}\right|_{E, T} \sigma_{k l}+\left.\frac{\partial \varepsilon_{i j}}{\partial E_{n}}\right|_{\sigma, T} E_{n}+\left.\frac{\partial \varepsilon_{i j}}{\partial T}\right|_{\sigma, E} T, \\
D_{m} & =\left.\frac{\partial D_{m}}{\partial \sigma_{k l}}\right|_{E, T} \sigma_{k l}+\left.\frac{\partial D_{m}}{\partial E_{n}}\right|_{\sigma, T} E_{n}+\left.\frac{\partial D_{m}}{\partial T}\right|_{\sigma, E} T, \\
S & =\left.\frac{\partial S}{\partial \sigma_{k l}}\right|_{E, T} \sigma_{k l}+\left.\frac{\partial S}{\partial E_{n}}\right|_{\sigma, T} E_{n}+\left.\frac{\partial S}{\partial T}\right|_{\sigma, E} T .
\end{aligned}
$$

Relevant differences have been underlined.

Considering the physical properties of the crystals, the symmetries of the tensor components and the order of differentiation of the thermodynamic function of state as a total differential, we can use a more concise matrix notation, e.g: 


$$
\begin{aligned}
& s_{i j k l} \rightarrow s_{m n} \quad(m, n=1, \ldots, 6), \text { fourth-order tensor, second-order physical } \\
& \text { constant; } \\
& s_{i j k l p q} \rightarrow s_{m n o} \quad(m, n, o=1, \ldots, 6), \text { sixth-order tensor, third-order physical } \\
& \text { constant; } \\
& d_{i j k l n} \rightarrow d_{m n o} \quad(m, n=1, \ldots, 6, o=1,2,3) \text {, fifth-order tensor, third-order } \\
& \text { physical constant; } \\
& d_{n i j} \rightarrow d_{n m} \quad(n=1,2,3, m=1, \ldots, 6) \text {, third-order tensor, second-order } \\
& \text { physical constant; } \\
& o_{i j n t} \rightarrow o_{m n t} \quad(m=1, \ldots, 6, n, t=1,2,3) \text {, fourth-order tensor, third- } \\
& \text { order physical constant; } \\
& \alpha_{i j} \rightarrow \alpha_{m} \quad(m=1, \ldots, 6) \text {, second-order tensor, second-order physical } \\
& \text { constant, etc. }
\end{aligned}
$$

During transition from tensor to matrix notation we neglected the coefficients that were different for relevant components (those for $s_{i j k l}$ and $s_{i j k l p q}$ are listed in section 3).

Equally concise are the matrices of the coefficients (tensor components) in nonlinear expansion. All of the coefficients (tensor components) incorporated in equations (2.6), (2.7) and (2.8), which occur on the right-hand side of table 1, are presented in the form of matrix $10 \times 62$ :

$$
(6+3+1) \times(6+21+15+6+3+6+3+1+1)=10 \times 62 .
$$

For linear effects we have matrix $10 \times 10$ :

$$
(6+3+1) \times(6+3+1)=10 \times 10 .
$$

Matrix notation is clear and very convenient, and in its external form it is similar for all (eight) thermodynamic functions, which have been used to describe the physical quantities for piezoelectric crystals. Making use of the data reported in the literature $[1,3,5,13,14,15,16]$, we can establish the number of independent components of particular tensors for each point group, as well as define the relations between some elements of these matrices.

\section{References}

1. Nye J.F. Physical Properties of Crystals. Oxford University Press, 1985.

2. Soluch W. // Wstęp do Piezoelektroniki. Wydawnictwo Komunikacji i Łączności, Warszawa, 1980 (in Polish).

3. Juretschke H.J. Crystal Physics. W.A. Benjamin, INC. Massachusetts, 1974.

4. Weber H.-J., Balashova E.V., Kizhaev S.A. // J. Phys.: Condens. Matter, 2000, vol. 12, p. 1485.

5. Fumi F.G., Ripamonti C. // Acta Cryst., 1980, vol. A36, p. 535.

6. Abrahams S.C. // Acta Cryst., 1994, vol. A50, p. 658. 
7. Briss R.R. Symmetry and Magnetism. Amsterdam, North-Holland Publishing Co., 1966.

8. Wooster W.A. Tensors and Group Theory for the Physical Properties of Crystals. 1973.

9. Warkusz F. // Zeszyty Naukowe UO, Fizyka, 1997, vol. 27, p. 195 (in Polish).

10. Mason W.P. // Phys. Rev., 1952, vol. 88, p. 480.

11. Mason W.P. Crystal Physics of Interaction Processes. New York, London, Academic Press, 1966.

12. Parton V.Z., Kudryavtsev B.A. Electromagnetoelasticity of Piezoelectronics and Electrically Conductive Solids. Moscow, Nauka Publ., 1988 (in Russian); Electromagnetoelasticity: Piezoelectrics and Electrically Conductive Solids. Taylor \& Francis, 1988 (English translation).

13. Fumi F.G. // Il Nuovo Cimento, 1952, vol. 9, p. 739.

14. Fieshi R., Fumi F.G. // Il Nuovo Cimento, 1953, vol. 10, p. 865.

15. Fumi F.G., Ripamonti C. // Acta Cryst., 1980, vol. A36, p. 551.

16. Fumi F.G. // Acta Cryst. 1997, vol. A53, p. 101.

\title{
Нелінійні механічні, електричні та термічні явища в п'єзоелектричних кристалах
}

\author{
Ф.Варкуш, А.Лінек \\ Інститут фізики, університет м. Ополє, 45-052 Ополє, Польща \\ Отримано 25 серпня 2002 р., в остаточному вигляді - \\ 31 березня $2003 \mathrm{p}$.
}

Механічні, електричні та термічні явища у п'єзоелектричних кристалах вивчаються у нелінійному наближенні. 3 цією метою використано термодинамічний потенціал, який описує анізотропне тіло. Розглянуто потенціал Гіббса. Розрахунки охоплюють тензор деформації $\varepsilon_{i j}=f\left(\sigma_{k l}, E_{n}, T\right)$, вектор індукції $D_{m}=f\left(\sigma_{k l}, E_{n}, T\right)$ та ентропію $S=f\left(\sigma_{k l}, E_{n}, T\right)$ як функцію механічного напруження $\sigma_{k l}$, величини поля $E_{n}$ і різниці температур $T$. Отримано рівняння, які описують анізотропні п'єзоелектричні тіла, якщо відомі “сили” $\sigma_{k l}, E_{n}, T$, що діють на кристал.

Ключові слова: п'єзоелектричні кристали, термодинаміка, анізотропні тіла, тензори

PACS: $65.40 .-b, 77.65 .-j$, 77.65.Bn, 77.65.Ly, 05.70.Ce 
